\title{
Tendenzen zum Monotheismus als Kennzeichen der religiösen Kultur der Spätantike Martin Wallraff
}

Aziz Al-Azmeb/János M. Bak (Hg.), Monotheistic kingship. The medieval variants (Central European University Medievalia 7 = Pasts incorporated 3), Central European University Department of Medieval Studies Budapest 2005, 297 S. - Polymnia Athanassiadi/Michael Frede (Hg.), Pagan Monotheism in Late Antiquity, Clarendon Press Oxford 1999, Nachdr. 2002, 211 S. - Loren T. Stuckenbruck/Wendy E.S. North (Hg.), Early Jewish and Christian Monotheism (JSNT.SS 263/Early Christianity in Context), Clark London 2004, VIII + 264 S.

Weitere Literatur:

Jan Assmann, Gesetz, Gewalt und Monotheismus: ThZ 62 (2006) 475-486. - Garth Fowden, Empire to Commonwealth. Consequences of Monotheism in Late Antiquity, University Press Princeton (New Jersey) 1993, XVII + 205 S. - Alfons Fürst, Monotheismus und Monarchie. Zum Zusammenhang von Heil und Herrschaft in der Antike: ThPh 81 (2006) 321-338. Alfons Fürst, Christentum im Trend. Monotheistische Tendenzen in der späten Antike: ZAC 9 (2006) 496-523. - Christoph Markschies, Heis Theos - Ein Gott? Der Monotheismus und das antike Christentum: Manfred Krebernik/Jürgen van Oorschot (Hg.), Polytheismus und Monotheismus in den Religionen des Vorderen Orients (AOAT 298), Ugarit-Verlag Münster

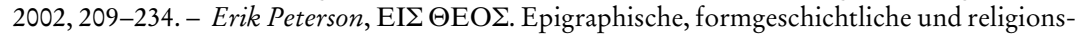
geschichtliche Untersuchungen (FRLANT 41), Vandenhoeck \& Ruprecht Göttingen 1926, VIII + 346 S. - Erik Peterson, Der Monotheismus als politisches Problem. Ein Beitrag zur Geschichte der politischen Theologie im Imperium Romanum [1935]: Erik Peterson, Ausgewählte Schriften, Bd. 1: Theologische Traktate, hg. v. Barbara Nichtweiß, Echter Würzburg 1994, 23-81. - Alfred Schindler (Hg.), Monotheismus als politisches Problem? Erik Peterson und die Kritik der politischen Theologie (Studien zur evangelischen Ethik 14), Gütersloher Verlagshaus Mohn Gütersloh 1978, 234 S. - Martin Wallraff, Viele Metaphern - viele Götter? Beobachtungen zum Monotheismus der Spätantike: Jörg Frey/Jan Rohls/Ruben Zimmermann (Hg.), Metaphorik und Christologie (TBT 120), Walter de Gruyter Berlin 2003, 151166. - Martin Wallraff, Pantheon und Allerheiligen. Einheit und Vielfalt des Göttlichen in der Spätantike: JAC 47 (2004) 128-143.

\section{Monotheistisches Christentum versus polytheistisches Heidentum?}

Christentum ist monotheistisch, und Heidentum ist polytheistisch. Dies ist die Standardsicht auf die Spätantike (und eigentlich alle Geschichtsepochen), ja, die gedankliche Verbindung von „Heidentum“ und „Polytheismus“ ist vielfach so fest „verdrahtet“, dass der zweitgenannte Begriff geradezu zu einem definitorischen Merkmal des erstgenannten werden kann. Es ist beinahe überflüssig zu erwähnen, dass der eine wie der andere begriffsgeschichtlich mit einer stark pejorativen Hypothek belastet ist: Beide Begriffe sind erfunden und zu dem Zweck in die Diskussion eingeführt, um sich von diesen negativ etikettierten Religionsformen abzugrenzen und um das Licht der jeweiligen Gegenbegriffe (Christentum/Judentum und Monotheismus) umso heller strahlen zu lassen. Beide Begriffe werden in der wissenschaftlichen Diskussion faute de mieux bis heute verwendet, doch ist es nicht einfach, sich von dem stark wertenden Beiklang freizumachen. Zur Beschreibung religionsgeschichtlicher Phänomene haben sie 
weiterhin einen gewissen heuristischen Wert, ja, sie bleiben vielfach alternativlos, jedenfalls für die Spätantike, selbst wenn die kruden Oppositionen aufgebrochen werden sollen (und in der jüngeren Diskussion vielfach auch aufgebrochen werden, dazu gleich mehr).

Die Gegenüberstellung von monotheistischem Christentum und polytheistischem Heidentum impliziert meist ein bestimmtes Geschichtsbild: einen Fortschrittsgedanken vom Sieg des Ersteren über das Letztere. Dieser Sieg ist - so die Auffassung - in der Spätantike errungen worden, und es ist bemerkenswert, wie ungebrochen sich dieses Geschichtsbild von den Zeitgenossen bis in heutige Lehr- und Handbücher gehalten hat. Denn es lässt sich zeigen, dass die genannte Gegenüberstellung, vor allem die Vorstellung vom polytheistischen Heidentum von bestimmten spätantiken Autoren verbreitet, ja eigentlich überhaupt erst gedanklich entwickelt, um nicht zu sagen: erfunden wurde (s. Fürst, Monotheismus, $321 \mathrm{f}$.; Wallraff, Pantheon, $128 \mathrm{f}$.). Es handelt sich um diejenigen Autoren der Zeit, von denen wir historisch am besten unterrichtet sind: der „intellektuelle Flügel“ des Christentums, also Kirchenväter und Theologen, die Sieger im Selektionsprozess des mittelalterlichen „Überlieferungsfilters“. Wenn diese Autoren von pagani sprechen, meinen sie die Bewohner des pagus, also ländlicher und zurückgebliebener Gebiete, die noch der überholten Vielgötterei anhingen. In diesem Sinne beerbt das deutsche Wort „Heidentum“ (in dem die „Heide“ steckt) die schlichten lateinischen pagani, nicht das theologisch reizvolle $\tau \dot{\alpha} \varepsilon ̋ \vartheta v \eta$ oder das vielschichtige oi ${ }^{\prime} E \lambda \lambda \eta v \varepsilon \varsigma$. Natürlich waren die Heiden in diesem wörtlichen Verständnis leichtere Gegner als die philosophisch gebildeten urbanen Eliten.

Indessen zeigt gerade die jüngere Diskussion der Forschung, dass die eingangs erwähnten Gleichsetzungen so einfach nicht funktionieren, dass also der monotheistische Charakter des Christentums und die Vielgötterei des Heidentums durchaus kritisch zu hinterfragen sind. Die christlichen Intellektuellen sind kritisch - mitunter auch „gegen den Strich“ - zu lesen, und vor allem sind sie durch andere Quellen zu ergänzen. Dabei ergibt sich ein erheblich differenzierteres Bild, das im Folgenden anhand einiger Beispiele aus der jüngeren Forschungsdiskussion vorgeführt werden soll. Zum Schluss soll noch einmal die Frage gestellt werden, welche Rolle der Monotheismus in der religiösen Kultur der Spätantike insgesamt spielte.

Hintergrund der Forschungsdebatte ist die insgesamt zunehmende Aufmerksamkeit, die das Thema Polytheismus und Monotheismus auf sich zieht, mitunter (wenn auch nicht immer) mit aktuellen Bezügen und Interessen. Durch solche Reflexionen fällt auch neues Licht auf die Religionsgeschichte der Spätantike. Natürlich ist der sachlich-quellenmäßige Befund vielfach nicht völlig neu und im Grunde den Spezialisten seit Langem bekannt. Auch wenn daher die Diskussion streckenweise alte Erkenntnisse nur plakativ neu verpackt (und daher gut verkauft), lässt sich doch mancherorts eine gedankliche Vertiefung feststellen, die eine eingehendere Beschäftigung lohnend erscheinen lässt. 
In der oben beschriebenen klassischen Perspektive wäre „paganer Monotheismus" eine contradictio in adiecto, eine Art schwarzer Schimmel, also etwas, das es nicht geben kann. Wer sich mit der Religionsgeschichte der Spätantike befasst, weiß natürlich, dass es so einfach nicht ist und dass monotheistische Tendenzen keineswegs nur innerhalb der Grenzen des Christentums und Judentums auftraten (und dass andererseits christliche und jüdische Lehre nicht in jedem Fall mit dem einfachen Label "Monotheismus" korrekt beschrieben ist). Es ist das Verdienst des von P. Athanassiadi und M. Frede herausgegebenen Buches, dass das Konzept des „paganen Monotheismus" nun zum Objekt eigener Reflexion wurde und damit geöffnet für weitere wissenschaftliche Diskussionen („Pagan Monotheism in Late Antiquity“, Oxford 1999 - die große Nachfrage nach diesem Buch zeigt sich an einem Nachdruck im Jahr 2002). Die sechs Beiträge in diesem Band umfassen sehr unterschiedliche religiöse Phänomene und auch sehr verschiedene historische Epochen. „Spätantike“ wird hier im weitestmöglichen Sinn verstanden, nämlich vom 2. vorchristlichen bis zum 7. nachchristlichen Jh. reichend (3) - und damit im Grunde sehr weit überdehnt.

Diese breite Definition ist nötig, um auch den Aufsatz „Towards Monotheism" von M. L. West (21-40) abzudecken, ein brillanter Essay zu den Ursprüngen dieser Vorstellung in der griechischen Philosophie. Es dauerte lange Zeit, bis das, was in der philosophischen Theorie entwickelt wurde, in die religiöse Praxis umgesetzt wurde - so die zentrale, durchaus überzeugende These. Vergleichsweise problematisch ist hingegen der folgende Artikel von M. Frede, ein Aufsatz von programmatischem Wert für das gesamte Buch. Frede zeichnet ein lebendiges und detailliertes Bild von „Monotheism and Pagan Philosophy in Later Antiquitiy“ (41-67, Spätantike hier verstanden im engeren, üblichen Sinn des Wortes). Es ist beeindruckend nachzuverfolgen, wie sich die verschiedenen philosophischen Schulen im Hinblick auf den theoretischen Monotheismus näher kommen, verstanden als Ein-Gott-Glauben (was natürlich eine Hierarchie niedrigerer Götter nicht ausschließt). In einigen Fällen wäre es allerdings hilfreich, wenigstens ansatzweise auf die zunehmende Ausprägung einer regelrechten religiösen Praxis einzugehen, vor allem bei den Philosophen platonischer Tradition (und daher praktisch aller spätantiken Philosophen, siehe den Beitrag von M. Perkams in diesem Heft, S. 50-64). Wenigstens in Bezug auf Julian wird diese pagane praxis pietatis im Beitrag von Athanassiadi behandelt (siehe unten).

Fredes gesamter Artikel zielt jedoch darauf ab zu zeigen, "that it is extremely difficult, if not impossible, to distinguish between the Christian position and the position of Plato, Aristotle, Zeno, and their followers in the later antiquity and thus the vast majority of philosophers in the late antiquity" (41). Die Nähe gewisser christlicher Positionen zu denen, die von manchen Philosophen vertreten werden, ist tatsächlich auffällig, auch schon oft konstatiert. Unglücklicherweise entspricht Fredes sehr differenziertem Bild der spätantiken Philosophie nicht ein 
ebenso differenziertes Bild der unterschiedlichen Strömungen und Gruppierungen innerhalb des Christentums. Es ist nicht sonderlich hilfreich zu sagen, dass "the Christians [were] willing, and even committed, to talk of a plurality of created beings as ,gods“" (60, meine Kursivierung). An dieser Stelle spricht Frede über einen Abschnitt in Augustins „De civitate Dei“ (9,23), wo der Autor feststellt, dass der größte Unterschied zwischen paganen Philosophen und der christlichen Lehre nicht im Gebrauch des Begriffs „Gott" für bestimmte unsterbliche Wesen liegt, sondern vielmehr in deren Rolle im Prozess der Rettung der Menschheit (also im Bereich der Soteriologie). Zweifellos ist der Text insofern erstaunlich, als er zeigt, dass tatsächlich auch im Christentum der Gebrauch des Wortes „Gott“ im Plural möglich ist: die Existenz der Götter wird nicht bestritten, sondern ihre soteriologische Relevanz. Auf der anderen Seite verweist er auf eine fundamentale Schranke, die in der christlichen Lehre nicht überwunden werden kann: die Schranke zwischen Schöpfer und Geschöpf. Geschaffene Wesen können nur vom ungeschaffenen Gott erlöst werden.

Im 5. Jh. ist dies vollkommen klar, besonders bei einem so scharfen Denker wie Augustin, doch war dem nicht immer so. Zu Recht weist Frede darauf hin, dass etwa Origenes' Christologie in dieser Hinsicht nicht völlig frei ist von gewissen Zweideutigkeiten (was vielleicht einer der Gründe ist, weshalb er bei späteren Generationen Misstrauen auslöste). Es ist kaum zufällig, dass eine der größten Krisen der christlichen Lehre in der Spätantike einer heftigen Debatte über eben diesen Sachverhalt entsprang: Der arianische (besser: trinitätstheologische) Streit kann als ein Prozess verstanden werden, der auf eine klare Grenzziehung zwischen Erlöser und Erlöstem, Schöpfer und Geschöpf zielte. Der Streit war so heftig und so schmerzhaft, weil die Debatte zwar mit philosophischen Termini geführt wurde, doch diese Grenze schließlich ohne die Hilfe der zeitgenössischen Philosophie, teilweise sogar ihr widersprechend, gezogen werden musste.

Natürlich ändert sich das Bild radikal, wenn man nicht nur die Reflexionen des christlichen Glaubens durch die intellektuelle Elite betrachtet, sondern auch die verschiedenen Formen christlicher Frömmigkeit in allen sozialen Schichten. Was für den Bischof von Hippo wahr ist, stimmt nicht notwendigerweise auch für einen christlichen Pilger auf dem Weg nach Jerusalem und noch weniger für einen Mönch in der ägyptischen Wüste. Die Quellenzeugnisse der magischen Papyri, Spuren des wachsenden Heiligenkultes und dergleichen sprechen eine völlig andere Sprache bezüglich Monotheismus und Polytheismus - Konzepten, die in diesem Zusammenhang sowieso mehr und mehr fraglich werden.

Diese Probleme werden in Fredes Darstellung nur marginal behandelt (64, mit eher summarischen Urteilen). Wenn christliche Prediger und Theologen auf dem theoretischen Monotheismus bestehen, wenden sie sich nicht notwendigerweise gegen pagane Gegner, sondern werden sich auch auf die Schäfchen aus ihrer eigenen Herde beziehen (man lese etwa Leo den Großen, tr. 22 Chavasse). Auch innerhalb des Christentums waren die Verhältnisse von großen Unterschieden gekennzeichnet. 
Andererseits verschmelzen die Identitäten des Christentums und der spätantiken Philosophie im Prozess der gegenseitigen Annäherung nicht völlig. Im Christentum wurden bestimmte Elemente des biblischen Erbes beibehalten, die einen „weichen“ Übergang vom Polytheismus zum Monotheismus unmöglich machten.

Dieses Problem mag sekundär scheinen, führt aber zu einer tiefer liegenden, teilweise sogar traumatischen Verletzung. Frede schlussfolgert: „It thus seems all the more puzzling why Christians insisted on their monotheism as a distinguishing mark." (67) Dies muss tatsächlich rätselhaft scheinen aus der Perspektive dessen, der nur den gemeinsamen Hintergrund beider Partner sieht, und nicht ihre jeweiligen differentiae specificae. Es ist bedauerlich, dass in dem Artikel viele wertvolle und wichtige Beobachtungen einer These untergeordnet sind, die sich ihrer eigenen Grenzen nicht bewusst ist.

In den übrigen Artikeln wird ein breites Spektrum unterschiedlicher Aspekte der Hauptthematik behandelt. Es ist nur natürlich, dass es in diesem schmalen Buch - wie in den meisten Sammelbänden - nicht möglich und auch nicht beabsichtigt war, das gesamte Feld systematisch abzudecken. Um gleich vorab einige derjenigen Aspekte zu nennen, die nicht oder kaum behandelt werden, obgleich sie wichtig wären: Es gibt einen kurzen und nicht sehr gründlichen Artikel zur gnostischen Tradition von J. Dillon (69-79). Auf die hermetische Tradition wird nur in der Einleitung kurz eingegangen (12f.), Mysterienkulte werden gar nicht erwähnt (vgl. 20 Anm. 49). Andere Aspekte erfahren eine ausgiebigere Behandlung.

Dies trifft im Besonderen auf die Verehrung des „höchsten Gottes“ (theos hypsistos) zu, die Gegenstand eines langen und sehr soliden Aufsatzes von S. Mitchell ist (81-148, ein Drittel des Buches!). Der Artikel beginnt mit einer Analyse der berühmten Inschrift an der Stadtmauer von Oenoanda, wo sich ein singuläres Zeugnis negativer Theologie findet: Gott ist „ohne Lehrer, ohne Mut-

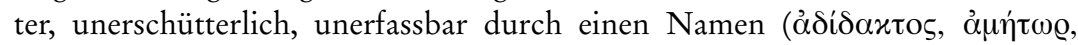

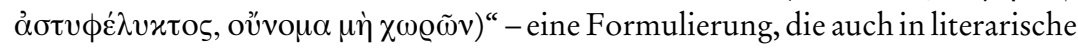
Texte übernommen wurde (Laktanz [div. inst. 1,7], Tübinger Theosophie [169 Erbse]). Mitchell behauptet, die Inschrift habe ihren Sitz im Leben im Kult des Theos Hypsistos. Diese These wird unterstützt von einer umfangreichen Materialsammlung anderer epigraphischer Quellen. Die Auflistung dieser Inschriften (128-147, fast 300 Stück) ist schon an sich von enormem Wert, da sie für jeden Forscher von Nutzen ist, der sich mit der schwierigen Frage des „Hypsistarianismus" auseinandersetzen will. Die Schwierigkeit bei der Beschreibung dieses Aspekts spätantiker Frömmigkeit besteht darin, dass er jeder präzisen terminologischen Beschreibung widerstrebt. Einerseits ist es nicht unbedingt angemessen, ihn als eigenständigen „Kult“ anzusehen, andererseits ist es falsch, „Hypsistos" nur als weiteren Beinamen anderer unabhängiger Götter zu betrachten. Einerseits steht er in gewissen Beziehungen zum Judentum, andererseits kann

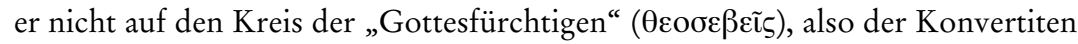


zum Judentum, reduziert werden. Nicht einmal das Geschlecht des höchsten

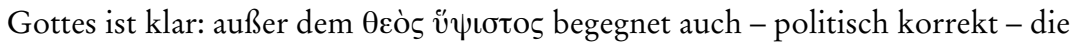

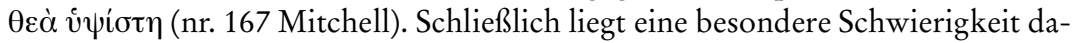
rin, dass das Phänomen zwar epigraphisch gut bezeugt ist, aber kaum literarische Hinweise darauf erhalten sind. Der einzige historisch bezeugte „Hypsistarier“ ist der Vater Gregors von Nazianz. Anlässlich seiner Bestattung gab sein Sohn Auskunft über dessen Glauben (or. 18) - nicht nur vom „häresiologischen“ Standpunkt aus in polemischer Perspektive, sondern sogar mit großem Wohlwollen. Doch selbst an dieser Stelle bleiben die Umrisse unscharf.

Vermutlich ist es angemessen, dem Phänomen eine gewisse Unschärfe zu belassen (in höherem Maße, als Mitchell dies tut) und den Begriff „hypsistarisch“ darum nicht allzu eng zu definieren. Er bezeichnet eher eine Tendenz als einen eigenen Kult. Wir wissen nichts oder beinahe nichts von einem besonderen Ritual oder speziellen Priestern dieses „Kultes“ (neuere Literatur bei Fürst, Christentum, 506f.). Der interessanteste und problematischste Aspekt im Kontext des Monotheismus ist nun allerdings die Frage, ob und in welchem Sinne die Anbetung des "höchsten Gottes“ monotheistische Tendenzen impliziert. Einerseits bedeutet die Verehrung eines höchsten Gottes nicht, dass die Existenz einer Vielzahl von Göttern bestritten wird. Andererseits vereint der höchste Gott die Namen und Charakteristiken verschiedener anderer Götter in sich, so dass er schließlich als einziger Gott stehen bleibt. Diese Konsequenz wird in den vorliegenden Texten jedoch nicht gezogen. Es wäre an dieser Stelle hilfreich - in Analogie zur alttestamentlichen Diskussion, wo dies schon seit langer Zeit üblich ist -, Konzepte wie Monolatrie oder Henotheismus in die Debatte einzubeziehen.

Ganz allgemein lässt sich sagen, dass die unscharfen Ränder, das programmatisch Diffuse des paganen Monotheismus in der Spätantike das Hauptproblem aller Forschungen auf diesem Gebiet ist. Abgesehen von einigen intellektuellen Christen und Juden beabsichtigte niemand einen kompletten Bruch mit den religiösen Traditionen der Antike. Aus diesem Grund gab es in vielen Fällen schlicht kein Interesse an einer klaren Abgrenzung zwischen Polytheismus und Monotheismus (bei denen es sich ja ohnehin um spätere Begriffsbildungen handelt) - und dies trotz einer faktisch weit verbreiteten Tendenz zum Monotheismus ab dem 3./4. Jh. Diese Sachlage erschwert die wissenschaftliche Beschreibung der Entwicklung sehr. Das beste und am weitesten fortgeschrittene Beispiel für diese Art des diffusen, ,in der Luft liegenden“ Monotheismus ist die Rede des Praetextatus in Macrobius' Saturnalien, die im letzten Beitrag des Bandes (W. Liebeschuetz; 185-205) genauer betrachtet wird. Es ist faszinierend zu sehen, wie in einem atemberaubenden Wirbel der Theokrasie alle Götter in die Sonne hineingezogen werden, in ihr aufgehen; so gut wie alles kann mit diesem letzten und höchsten Gott der paganen Spätantike identifiziert werden.

P. Athanassiadis Abhandlung über die chaldäischen Orakel (149-183) ist im Grunde ein Beitrag zur Literaturgeschichte des spätantiken Neuplatonismus 
und daher nur oberflächlich mit dem Generalthema „Monotheismus“ verbunden. Immerhin tritt in dieser Literaturgruppe eine Art praxis pietatis der paganen Philosophie vor Augen, eine Art „cult to match a more spiritual conception of the divine“ (178). Die Orakel zeigen, dass es auch „a more spiritualized form of piety than the one associated with blood sacrifices“ (180) gab. Kurz gesagt: Die Orakel waren Teil des „late antique spiritual Commonwealth“. Ob der mitunter inflationäre Gebrauch der Termini „spirituality“, „spiritual“ usw. zur Klärung beiträgt, bleibe dahingestellt. In der Sache ist der Hinweis wichtig und richtig, dass das so genannte „Heidentum“ auch im Bereich der Frömmigkeit ein enormes Potenzial für religiöse Innovationen aufwies und dass die Orakel ein hervorragendes Zeugnis dafür darstellen.

In ihrer allgemeinen Einführung meinen die Herausgeber: „Christianity did not convince because it was monotheistic; rather it would appear that in order to convince, it had to be monotheistic in a society which was fast moving in that direction." (20) Das ist sicherlich wahr. Andererseits ist es genauso wahr zu sagen, dass das Heidentum monotheistisch sein musste, um zu überzeugen. Es ist ausgesprochen schwierig, wahrscheinlich sogar unmöglich, festzustellen, was zuerst kam, was Ursache und was Wirkung war: der Monotheismus als solcher oder konkrete Religionsformen, die ihn vertraten. Es ist das Verdienst dieses Buches, die Frage neu gestellt und in einigen Fallstudien detailliert vor Augen geführt zu haben.

\section{Monotheismus und Monarchie - die politischen Konsequenzen}

Eine der sensibelsten und brisantesten Fragen, die sich mit der monotheistischen Tendenz verbindet, ist die nach den politischen Konsequenzen - bei Athanassiadi/Frede ganz ausgeblendet (doch siehe schon früher Fowden). Die Frage ist so schwierig, weil sie fast immer auch mit systematischen Interessen gestellt wird, also zum Prinzipiellen neigt: Setzt der Monotheismus im religiösen Bereich automatisch auch entsprechende Formen staatlicher Organisation frei, entsprechen ihm also notwendigerweise absolute Monarchie und Diktatur? Vollends brisant wird die Frage, wenn sie mit Gegenwartsbezug gestellt wird, und das war bekanntlich der Fall bei Erik Petersons Klassiker „Der Monotheismus als politisches Problem“, erschienen 1935, also in der Anfangsphase der nationalsozialistischen Diktatur in Deutschland. Es ging dabei um die Spätantike, doch gleichzeitig um mehr. G. Geréby hat dies in einem feinsinnigen Aufsatz jüngst eindrucksvoll herausgearbeitet.

Der Aufsatz ist zu lesen in einem insgesamt zu wenig beachteten ungarischen Tagungsband über „Monotheistic Kingship“, der die Frage nach Monotheismus und Monarchie allgemein stellt, allerdings mit Schwerpunkt auf dem Mittelalter, wie der Untertitel sagt („The Medieval Variants“). Die Tagung fand im Februar 2002 in Budapest statt, organisiert von A. Al-Azmeb und J. M. Bak, die auch den Band herausgegeben haben. Der theoretische Ansatz wird im einführenden Auf- 
satz von A. Al-Azmeh (9-29) erklärt. Der Ausgangspunkt ist zunächst nicht die Monotheismus-Debatte, sondern das Konzept des sakralen Königtums. Freilich ist damit die Beziehung des Herrschers zum Göttlichen immer schon mit im Blick, und eine der denkbaren Varianten ist eine Legitimationsachse von dem einen Gott zu dem einen Herrscher. Al-Azmeh bezieht sein historisches Belegmaterial aus einem weit gefächerten Spektrum: von den Anfängen der angelsächsischen Kultur in England bis zur Frühgeschichte des Kalifats in Damaskus. Doch eine herausgehobene Rolle spielt das römische Reich in der Spätantike (15-22). In dieser Kultur wird ein „pronounced henotheistic streak“ diagnostiziert, der schließlich zum regelrechten Monotheismus führte (15). Al-Azmeh sieht in der Spätantike das Erbe der Antike kulminieren, indem sich die Tendenzen zum Monotheismus, Absolutismus und Universalismus in einer großen zentralen politisch-religiösen Weltanschauung treffen (18); dieser stehen freilich stets diverse zentrifugale Tendenzen gegenüber. Manche Schlussfolgerungen in dem weit ausgreifenden Aufsatz gehen etwas schnell, aber anregend ist er allemal. Besonders hervorzuheben ist die Souveränität, mit der die Herrschaftskonzepte des frühen Islam aus der Spätantike abgeleitet werden, nicht einfach ihr in kruder Opposition gegenübergestellt.

Unter den folgenden Aufsätzen ist der substanziellste und für das hier betrachtete Thema einschlägigste der schon genannte Beitrag von G. Geréby über die politische Theologie bei Carl Schmitt und Erik Peterson (31-61). Der Beitrag gibt sich im Untertitel bescheiden als „a footnote to Kantorowicz“, doch für eine Fußnote ist er mit über 30 Seiten entschieden zu lang, und auch der gedankliche Gehalt geht weit über eine Marginalnotiz hinaus (doch Fußnoten spielen in dem Aufsatz eine erstaunlich große Rolle!). Der Verfasser möchte neues Licht auf den Zusammenhang zwischen Schmitts Theorie einer „politischen Theologie“ und Petersons Monotheismus-Buch werfen. Erst im Verständnis dieser Zusammenhänge - so die These - erschließt sich sodann der Hintergrund der epochalen Studie „The King's Two Bodies“ von E. H. Kantorowicz (1957). Die historische Rekonstruktion dieses Dreiecks (Schmitt - Peterson - Kantorowicz) ist nicht einfach, weil die wechselseitigen Bezugnahmen oft eher implizit und indirekt sind, dafür aber angesichts der politischen Verhältnisse von höchstem historischem Interesse.

Der Jurist Schmitt und der Theologe Peterson, die für einige Zeit unmittelbare Kollegen an der Universität Bonn waren und sich von dort gut persönlich kannten (Peterson war Trauzeuge bei Schmitts zweiter Eheschließung!), sind biographisch und geistig später höchst unterschiedliche Wege gegangen. Während der eine seine Bonner Fakultät und die evangelische Kirche verließ und als katholischer Konvertit den Rest seines Lebens unter materiell schwierigen Bedingungen in Rom verbrachte, ging der andere nach Berlin und rückte dort zum Ratgeber Görings und zum Vorsitzenden der Vereinigung nationalsozialistischer Juristen auf. Natürlich darf man Schmitts Antrittsvorlesung von 1924, in der er seinen Begriff von „politischer Theologie“ prägte, nicht nur im Licht der späte- 
ren Ereignisse lesen, aber Petersons Reaktion ist nicht ohne diese Ereignisse denkbar. Geréby weist mit Recht auf die letzte Fußnote in Petersons Buch hin, die als Schlüssel für das Gesamte dienen kann: „Der Begriff der ,politischen Theologie' ist $\mathrm{m}$. W. von Carl Schmitt [...] in die Literatur eingeführt worden. [...] Wir haben hier den Versuch gemacht, an einem konkreten Beispiel die theologische Unmöglichkeit von ,politischer Theologie‘ zu erweisen." (zitiert 35, bei Peterson, Monotheismus, 81 Anm. 168)

Zugrunde liegt also Schmitts Säkularisierungshypothese, der zufolge das neuzeitliche politische Denken nur aus seinen theologischen Wurzeln zu verstehen ist, also eine Transformation des Theologischen in den Bereich des Staatsdenkens darstellt. Auch in seiner säkularen Form setzt es stets eine letztlich nicht immanent begründbare Staatsgewalt voraus, und in Schmitts Denken kann diese nur als Einheit gedacht werden, so dass die Wurzel des Gedankens im strengen Monotheismus der christlichen Tradition liegt. 1924 wird noch eher beiläufig und indirekt darauf hingewiesen, dass nach dem Ende der Monarchie als einzige politische Alternative die Diktatur bleibt (Al-Azmeh/Bak, 40). Natürlich gewannen solche Aussagen in den 30er Jahren neue Brisanz. Petersons Antwort ist wesentlich eine aktuelle Auseinandersetzung im Medium der historischen Rekonstruktion der spätantiken Religionsgeschichte.

Schon früher war ihm im Rahmen seiner Habilitationsschrift „EI $\Sigma \Theta E O \Sigma$ “ (1926) fraglich geworden, inwieweit die zahlreichen epigraphischen Zeugnisse mit der Ein-Gott-Formel wirklich als Belege für Monotheismus oder auch nur eine Vorstufe dazu gelten können. Die Formel will keine Aussage über die Einheit oder Einzigkeit Gottes machen, sondern hat Bekenntnischarakter im religiösen wie im politischen Bereich („mein Gott ist einzigartig/souverän“). Wenn man von hier aus nun die weiter gehende Frage stellt, wie sich das orthodoxe Christentum des 4. Jh. zu solchen Theologoumena verhält, so kommt dessen Widerständigkeit gegenüber politischen Vereinnahmungen zum Vorschein. Peterson möchte zeigen, dass gerade die trinitätstheologischen Streitigkeiten ein Ergebnis zeitigten, das jeder politischen Funktionalisierung der Theologie einen Riegel vorschob. Zugespitzt formuliert: „What he is going to prove is that Christianity in its normative form, is not monotheism“ (47). Als Kronzeugen hierfür werden vor allem die drei großen Kappadokier aufgerufen.

Diese Beobachtungen von Geréby sind nicht völlig neu; ebensowenig die Diskussion, ob Petersons Rekonstruktion diesen historischen Quellen gerecht wird (siehe etwa den von Schindler herausgegebenen Band). Wichtig ist nun aber der Hinweis, dass die Botschaft nicht völlig ungehört verhallte. Geréby kann zeigen, dass Schmitt zumindest auf dem Wege über J. Taubes, mit dem er in Kontakt stand, auf die versteckte Kritik aufmerksam wurde. Die einschlägigen Briefzitate sind in dieser Hinsicht äußerst aufschlussreich (49f.). Die eigentliche Pointe in Gerébys Aufsatz ist zum Schluss der Bezug zu Kantorowicz. Er macht plausibel, dass die Schmitt-Peterson-Debatte in dessen Studie „The King's Two Bodies“ implizit präsent ist - und zwar bemerkenswerterweise so, dass Kantorowicz die 
Gedankenfigur Schmitts im Prinzip übernimmt, aber kritisch gegen das Christentum wendet. Dabei wird Schmitt mit keinem Wort genannt - nach Geréby „a case of damnatio memoriae" (54).

Die feinsinnige Rekonstruktion einer intellektuellen Debatte von vor mehr als einem halben Jahrhundert hat wichtige Implikationen sowohl für die Beschäftigung mit der Spätantike generell als auch für heute aktuelle Debatten. Geréby verzichtet darauf, Verbindungslinien zu den jüngeren Diskussionen über Monotheismus und Gewalt sowie über das Konzept einer „politischen Theologie“ etwa bei J. Assmann zu ziehen (siehe lediglich, knapp, 40). Solche Linien ließen sich leicht ziehen und sollten auch gezogen werden - und sei es nur, um zu verhindern, dass sich die Diskussion im Kreis dreht und längst ausgefochtene Kämpfe wieder aufnimmt.

Nur knapp sei auf zwei weitere Aufsätze im Band über „Monotheistic Kingship“ verwiesen, die von Interesse für die Erforschung der Spätantike sind. C. Gapar spricht über „The King of Kings and the Holy Men“, das Verhältnis der königlichen Autorität und der charismatischen Macht der Heiligen im frühen Byzanz (63-88). In Terminologie und Methodik stark an P. Brown orientiert, untersucht er einige Texte, in denen sich die - scheinbar unumschränkte Macht des Monarchen dem Rat und dem Einfluss eines „Holy Man“ unterordnet. Dabei kann er zeigen, dass dieses Charisma keineswegs auf christliche Heilige beschränkt ist, auch wenn dort natürlich die Quellen reicher fließen. Der „Holy Man“ als solcher zieht eine bislang in der Antike nicht gekannte Aufmerksamkeit auf sich, die sich keineswegs nur aus dem individuellen Streben nach geistlicher Perfektion erklären lässt. Darin spiegelt sich eine kollektive Sehnsucht nach dem „religiösen Genie“, nicht selten gekoppelt mit einer Art geistlicher „Schausucht“. Mit Monotheismus hat all dies zunächst nicht viel zu tun, doch für den politischen Herrscher folgt daraus einerseits eine Beschränkung der Macht des Monarchen, andererseits nochmals von einer anderen Seite eine Bestätigung für die typisch spätantike sakrale Grundierung der politischen Macht (und zwar nicht speziell und ausschließlich als Folge des Christentums!).

Dieses Motiv liegt auch dem Beitrag von I. Karaulashvili zugrunde (173-190): es geht um die Abgar-Legende, derzufolge Jesus mit dem damaligen König von Edessa (Abgar Ukkama bar Manu) einen Briefwechsel unterhalten habe. Diese weit verbreitete Legende wurde ab dem 6. Jh. von den Königen von Edessa gezielt zur Herrschaftslegitimierung eingesetzt. Schon vorher wurde dem Brief Jesu apotropäische Funktion zugeschrieben: Seine Abschriften helfen bei der Verteidigung der Stadt. Für das Monotheismus-Thema ist nun die Ergänzung um das Bild Christi von größter Bedeutung. Generell lässt sich in justinianischer Zeit feststellen, dass der Monarch als imago Christi erscheint und daher seine Legitimität durch den Besitz eines „echten“ Christus-Bildes steigern kann. In Edessa verehrte man seit dieser Zeit eine „nicht von Händen gemalte Ikone“, die angeblich von Christus selbst übersandt worden sein soll, das (später so genannte) Mandylion (178). Die irdische Monarchie bildet auf diese Weise die 
himmlische für jedermann sichtbar ab. Der hauptsächliche innovative Wert des Aufsatzes liegt indessen in der Behandlung der Wirkungsgeschichte dieses Überlieferungskomplexes im hohen Mittelalter in Georgien (184-190). So interessant dies auch ist - das Referat sei hier abgebrochen, weil die zeitlichen Grenzen der Spätantike damit weit verlassen sind.

\section{Monotheismus in Christentum und Judentum}

Auch der nächste anzuzeigende Band liegt zeitlich gesehen am Rande der hier relevanten Zeit, doch während der vorausgehende am oberen Rand - zum Mittelalter hin - lag, geht es hier um die Frühgeschichte in hellenistischer Zeit, speziell um die Zeit und die Schriften des Neuen Testaments. Auch diesem Band kann daher die Besprechung nicht umfassend gerecht werden, sondern nur, soweit er Anregungen für die Religionsgeschichte der Spätantike bietet. Und solche Anregungen werden durchaus geboten! Unter dem Titel „Early Jewish and Christian Monotheism" haben L. T. Stuckenbruck und W. E. S. North dreizehn Beiträge publiziert, die ursprünglich als Vorträge in einem Postgraduate Seminary an der Universität Durham gehalten wurden.

Die Herausgeber legen einleitend über die Fragestellung und das Interesse Rechenschaft ab. Untersucht werden die „contours of early Christian beliefs about Jesus in relation to the one God of Israel“ (1). Implizite Voraussetzung für diese Frage ist, dass die diversen frühen Christologien in mehr oder minder ausgeprägter und ausdrücklicher Weise den tradierten Monotheismus des Judentums in Frage stellen, um nicht geradezu (viel pejorativer) zu sagen: ihn gefährden. Im Verein mit dem gleich zu Beginn bekundeten Interesse, nicht nur eine historische Rekonstruktion vorlegen, sondern auch dem „interfaith (especially Jewish-Christian) dialogue“ (1) dienen zu wollen, entsteht eine potenziell problematische Gemengelage: Die verwendeten Begriffe und vorgefassten Interessen könnten störend mit dem Zeugnis der Quellen interferieren und letztlich keine neuen Erkenntnisse zutage fördern, sondern nur in leicht verfremdeter Gestalt das, was womöglich ohnehin den Ausgangspunkt des Unternehmens bildete (nämlich - um es direkt und zugespitzt zu sagen - eine politisch korrekte „Weichspülerchristologie").

Schon der erste Beitrag macht indes deutlich, dass diese Sorge nicht oder jedenfalls nicht durchgängig begründet ist. Der Cambridger Judaist W. Horbury untersucht "Jewish and Christian Monotheism in the Herodian Age" (16-44) und plädiert dabei für einen differenzierten Gebrauch des Begriffs Monotheismus bei der Beschreibung jüdischer religiöser Identität im herodianischen Zeitalter. Der Begriff sei nicht rundheraus abzulehnen, doch in unterschiedlichen Kontexten und Epochen eher „inklusiv“ oder „exklusiv“ zu nuancieren. Das herodianische Zeitalter sei - so die These - durch einen „inklusiven Monotheismus" gekennzeichnet, der erst später, vor allem in der rabbinischen Literatur, durch stärker exklusive Tendenzen verdrängt wurde: Dieser zweitgenannte Mo- 
notheismus ist es, der das Bild des Judentums weithin bis heute bestimmt. Mit souveräner Quellenkenntnis zeigt Horbury, wie Ausdrücke aus der Tradition der hebräischen Bibel im herodianischen Zeitalter aufgenommen, aber später als anstößig ausgeschieden werden: der "Gott der Götter" (Dtn 10,17), der „große König über allen Göttern“ (Ps 95,3), der „Führer der Geister“ (2 Makk 3,24) und dergleichen (20f.). Es gibt keinen Grund anzunehmen, dass das entstehende Christentum an dieser inklusiven Tendenz nicht genauso Anteil hatte wie an der exklusiven (die durchaus daneben auch existieren konnte). Die Analyse der jüdischen und christlichen Apologetik zeigt, dass die Verwerfungslinien hier anders und komplizierter laufen als in der Opposition Mono- versus Polytheismus. Horbury macht sogar auf einen wenig beachteten Aspekt des inklusiven Monotheismus aufmerksam, der auf ein erstaunlich weitgehendes Maß jüdischer Akzeptanz paganer Religionsformen hinauslaufen konnte - jedenfalls soweit man in ihnen Anschlussstellen für die monotheistische Tendenz erkennen konnte (27-31). Dass in diesem Aufsatz ein forschungsgeschichtlicher Rückblick erst ganz am Schluss erfolgt, ist ungewöhnlich, aber hier sehr sinnvoll: Erst auf der Basis der quellengeleiteten Rekonstruktion wird deutlich, in welchem Maße die Kategorien moderner Forschung Fragestellungen und damit mögliche Resultate prägen (in diesem Falle die einflussreichen Arbeiten von W. Bousset und G. F. Moore).

Der Aufsatz von Horbury trägt nicht nur im historischen Gehalt, sondern auch durch die klare Begriffsbildung zum Verständnis weit über das herodianische Zeitalter hinaus bei. Die Spätantikenforschung tut gut daran, hier genau zuzuhören und für das eigene Feld zu lernen. Die folgenden Beiträge mit primär exegetischem Interesse seien hier übergangen, um direkt zu den beiden abschließenden Aufsätzen zu kommen, die wiederum für das begriffliche Rüstzeug von Interesse sind. N. MacDonald untersucht „The Origin of ,Monotheism “" (204-215, die Studie ist aus MacDonalds Monographie hervorgegangen: „Deuteronomy and the Meaning of ,Monotheism“", Tübingen 2003). MacDonald geht von der zutreffenden Einsicht aus, dass den Begriff Monotheismus heute nur angemessen gebrauchen kann, wer ihn im Blick auf seine historischen Wurzeln befragt hat. Diese Wurzeln liegen bekanntlich nicht in der Zeit der hier relevanten Quellentexte; es handelt sich vielmehr um eine neuzeitliche Prägung. Die begriffsgeschichtliche Reise beginnt mit den Cambridge Platonists im 17. Jh., im Speziellen mit H. Moore. Dort begegnet das Wort allerdings zunächst in einem Sinn, der dem modernen "Pantheismus“ näher ist (205-210). Der heutige Gebrauch ist erst im 19. Jh. entstanden, und zwar - so die These - stets im Zusammenhang mit bestimmten historischen Entwicklungsmodellen, also zur Bezeichnung einer Phase in einem Aufstiegsoder Abstiegsgeschehen (213-215). In diesem Zusammenhang sind auch die Termini Henotheismus und Monolatrie entstanden - als unvollkommene Vorstufen zum „eigentlichen“ Gipfel, dem Monotheismus. Wie in manchen anderen Fällen auch spielt auch hier der fatale M. Müller eine Schlüsselrolle: Seine 
religionshistorischen Theorien sind materialiter durchweg heute nicht mehr state of the art, aber die von ihm geprägte Begrifflichkeit ist - oft eher unbewusst - zur wissenschaftlichen koine geworden. Eine eingehende Beschäftigung würde also lohnen - auch über die knappen Bemerkungen von MacDonald hinaus. Die Auseinandersetzung mit den antiken Quellentexten tut gut daran, zunächst zu untersuchen, wonach man eigentlich fragt, wenn man nach Monotheismus fragt. Nicht dass die Frage an sich nicht legitim wäre - sie bedarf nur der methodischen Reflexion.

Während MacDonald hieran nicht zweifelt, tritt der letzte Aufsatz mit einem anderen Anspruch auf (R. W. L. Moberly; 216-234). Die Frage im Titel „How appropriate is ,Monotheism' as a Category for Biblical Interpretation?" gibt sich leicht als rhetorisch zu erkennen. Die Begriffsgeschichte in Aufklärung und Historismus wird hier als „Entlarvung“ präsentiert. Daraus resultiert, dass diese Terminologie der "Classical Western Christian Theology" (232) aufgegeben werden muss. An deren Stelle sollen „nuanced and revisable interpretative categories" treten, die freilich nicht weiter spezifiziert werden (233). Indes: Dass auch die kritisierte „Western theology" mit revidierbaren Begriffen arbeitet und arbeiten muss, wenn man ihr nicht a priori sklerotische Erstarrung im terminalen Stadium unterstellt, dürfte klar sein. Umgekehrt wird der Leser den Verdacht nicht los, dass der Versuch, unter Umgehung der Aufklärung direkt zur Bibel „zurückzuspringen“, nicht in biblischer, sondern biblizistischer Theologie wurzelt. Dies freilich ist nicht der Gesamtduktus eines Buches, das einen differenzierten und nuancierten Beitrag zur altertumswissenschaftlichen Debatte über den Monotheismus leistet.

\section{Ausblick auf aktuelle Forschung}

Der Überblick zeigt, dass die Frage nach dem spätantiken Monotheismus in jüngerer Zeit wieder verstärkt das Interesse der Forschung findet (s. auch die Beiträge von Markschies, Wallraff und Fürst). Abschließend sei auf einige aktuelle Vorhaben aufmerksam gemacht, die unmittelbar vor dem Abschluss stehen und die die Kenntnis des Phänomens wesentlich erweitern werden. Für die Vorgeschichte und den Hintergrund ist die neue große Monographie von O. Keel über die "Geschichte Jerusalems und die Entstehung des Monotheismus" relevant (im Druck bei Vandenhoeck \& Ruprecht). Ebenfalls kurz vor der Publikation steht der kommentierte Nachdruck von Erik Petersons Klassiker „EI $\Sigma$ $\Theta E O \Sigma$ “ in den „Ausgewählten Schriften“ dieses Autors, besorgt von B. Nichtweiß. Der Band wird von Ch. Markschies gestaltet und verantwortet; er wird sowohl für die Wissenschaftsgeschichte als auch für die Erforschung des spätantiken Inschriftenmaterials höchst aufschlussreich sein.

Den umfangs- und gehaltsmäßig wichtigsten Zuwachs kann sich die Forschungsdiskussion von einer Tagung im Juli 2006 versprechen: eine große Zahl von Wissenschaftlern unterschiedlicher Disziplinen aus aller Welt diskutierte in 
Exeter über „Pagan Monotheism in the Roman Empire $\left(1^{\text {st }}-4^{\text {th }} \text { cent. }\right)^{\text {“. }}$. Der Ansatz ist, wie der Titel verrät, dem kleinen Buch von Athanassiadi/Frede verwandt, doch hier nun viel weiter gespannt. Die zahlreichen Beiträge decken ein breites Spektrum ab, sowohl die behandelten Quellen betreffend - von den Apollo-Orakeln (A. Busine) bis zur Varro (G. van Kooten) - als auch in Bezug auf die Religionsgemeinschaften - vom Dionysos-Kult (E. Kessler) bis zu Philo und anderen jüdischen Gruppen (H. Clifford, L. Rutgers, wobei das Epitheton „pagan“ an seine Grenzen kommt). Ein erkennbarer Schwerpunkt besteht allerdings in der neuplatonischen Philosophie, und das ist wohl mehr als Zufall. Die Organisatoren der Tagung, S. Mitchell und P. van Nuffelen, sind dabei, die Vorträge zum Druck vorzubereiten. Man darf auf das Ergebnis gespannt sein!

\section{Religionsgeschichtlicher Ertrag}

Die jüngere Debatte hat gezeigt, dass die Opposition von „monotheistischem Christentum“ und „polytheistischem Heidentum“, wie sie von den Kirchenvätern suggeriert wurde und in den Handbüchern lange fortlebte, zur Erfassung der religionsgeschichtlichen Zusammenhänge in der Spätantike nicht geeignet ist. Weder ist das Christentum in allen seinen Ausformungen und Gruppierungen mit der Etikette „Monotheismus“ ausreichend beschrieben, noch enthält der bunte Blumenstrauß des „Heidentums“ ausschließlich (oder selbst nur: vorwiegend) polytheistische Religionsformen oder -tendenzen. Vielmehr ist deutlich, dass in beiden Fällen genauer differenziert werden muss, ja es ist fraglich, ob das bipolare begriffliche Instrumentarium des Polytheismus und Monotheismus überhaupt zur Beschreibung ausreicht. In dieser Feststellung stimmen die meisten Autoren durchaus überein (zuletzt Fürst, Christentum, 497), doch ist damit allein noch nicht viel gewonnen. Von der Diagnose des Defekts zur angemessenen Therapie ist es ein weiter Weg.

Mit Recht wird mitunter gefordert, die in anderen Disziplinen längst eingeführten Begriffe Henotheismus und Monolatrie auch für die Spätantike fruchtbar zu machen. Damit ließen sich möglicherweise Phänomene wie die Verehrung des „Theos Hypsistos“ oder der Aufstieg des ägyptischen Sarapis zur „Leitgottheit“ besser beschreiben. Das auffälligste Phänomen in der Spätantike entzieht sich indes auch dieser Kategorisierung. Denn der eine Gott der Spätantike geht oft nicht so sehr aus dem eifersüchtigen Ausstechen der konkurrierenden Götter hervor, sondern eher aus einem etwas unübersichtlichen Prozess der Theokrasie. Allenthalben begegnen Götter mit unklaren Identitäten, unscharfen Rändern, Götter, die - mir nichts, dir nichts - von Zeus zu Sol und von Sol zu Christus changieren. So unübersichtlich diese Situation auch ist - es herrscht nicht einfach Beliebigkeit. Zur genaueren begrifflichen Differenzierung sei daher die Rede von inklusivem und exklusivem Monotheismus empfohlen (im Anschluss etwa an den genannten Horbury).

Inklusiv ist die monotheistische Tendenz dort und so lange, wie sie weitere 
Götter durch Theokrasie in sich aufzunehmen bereit ist. Es würde beispielsweise erkennbar werden, dass die Religionspolitik Kaiser Konstantins durch einen sehr ausgeprägten inklusiven Monotheismus geprägt war. In diese monotheistische Reichsreligion sollte nicht zuletzt das Christentum integriert werden. Indessen kam es hier alsbald zu Problemen: das Christentum selbst wollte so nicht mit sich umgehen lassen, es erkannte und betonte Grenzen, vertrat insofern einen eher exklusiven Monotheismus. Wo genau lagen die Grenzen der Theokrasie? Es wäre lohnend, die Quellen der Zeit noch einmal auf diese Frage hin sprechen zu lassen.

Das jüngere Interesse am spätantiken Monotheismus zeichnet sich ein in ein generelles Interesse am Thema, gespeist aus aktuellen Erfahrungen und Reflexionen. Dabei dürften vor allem zwei Gesprächskonstellationen relevant sein. Zum einen: Monotheismus als verbindendes Element in einer interreligiösen communio abrahamitischer Traditionen, also als Klammer über Judentum, Christentum und Islam. Zum anderen: Monotheismus und Gewalt. In dieser Perspektive unterliegt der Monotheismus mitunter einem Generalverdacht als Wurzel von religiöser Intoleranz und Fundamentalismus (zuletzt etwa Assmann). Die jüngere Forschungsdiskussion zur Spätantike bietet für beide Fragestellungen anregendes Material, doch mahnt sie auch zur Vorsicht. Insbesondere die Einsicht, dass Christentum und Monotheismus nicht einfach gleichzusetzen sind, lässt fraglich erscheinen, ob das vom Christentum ausgehende Gewaltpotenzial oder die vom Christentum erfahrene Gewalt jeweils ihre Hauptursache in monotheistischen Glaubensformen haben. Ebenso zeigt sich, dass der Islam in mancher Hinsicht als Fortführung und Radikalisierung von monotheistischen Tendenzen verstanden werden kann, wie sie schon das spätantike Christentum (und Judentum) aufweisen - wiederum mit keineswegs eindeutigen Folgen für das Phänomen Gewalt.

Abschließend noch einmal die von Frede aufgeworfene Frage: Ist die spätantike Tendenz zum Monotheismus Ursache oder Folge für das Aufkommen bestimmer religiöser Phänomene im römischen Reich (Christentum, Judentum, Neuplatonismus)? Es ist wie die Frage nach der Henne und dem Ei. Es gibt Phänomene in der Geschichte der Menschheit, die in Erscheinung treten, ohne dass man den einen genauen Grund dafür angeben kann. Sehr wohl aber lassen sich Gründe benennen. Ob das Christentum als „Trittbrettfahrer“ dieser Strömung des Zeitgeistes groß geworden ist oder ob es diese Strömung allererst (mit) hervorgebracht hat, kann dabei bis zum gewissen Grad offen bleiben. 